American Journal of Applied Sciences 1 (3): 255-257, 2004

ISSN 1546-9239

(C) Science Publications, 2004

\title{
On a Loss of Information in a Transition from Quantum to a Quasi-Classical Regime
}

\author{
Alex Granik \\ Department of Physics, U.O.P, Stockton, CA.95211, USA
}

\begin{abstract}
By defining information entropy in terms of the probability density $|\psi|^{2}$ ( $\psi$ is a wave function in the coordinate representation) it is explicitly shown how a loss of quantum information occurs in a transition from a quantum to a quasi-classical regime.
\end{abstract}

Key words: Information, Entropy, Quantum to Classical Transition, Loss of Information

\section{INTRODUCTION}

The Boltzmann-Gibbs-Shannon entropy:

$I=-\sum_{i=1}^{N} p_{i} \log p_{i}$

( $\mathrm{p}_{\mathrm{i}}$ is the classical probability) has an obvious relation to von Neumann entropy:

$S=-\operatorname{Tr}(\rho \log \rho)$

where the diagonal terms of the density matrix rij are the probabilities $\mathrm{p}_{\mathrm{i}}$.

Usually in quantum mechanics the probability $\mathrm{p}_{\mathrm{i}}$ is considered as a function of energy $E_{i}^{[1]}$. Since we study a rather narrow problem of information dynamics when the probability is described in terms of the wave function $\psi$, we will not discuss a more sophisticated (and complete) approach based on the density matrix and information processing involving quantum bits (qubits) ${ }^{[2]}$.

To achieve our goal, we proceed along the lines used in derivation of continuity equation for probability density. To this end consider the probability in its coordinate representation. Thus pi can be viewed as a probability of finding a particle in a spatial interval:

$\Delta \mathrm{q}_{\mathrm{i}}=\mathrm{q}_{\mathrm{i}+1}-\mathrm{q}_{\mathrm{i}}: \mathrm{p}_{\mathrm{i}}==\int_{\mathrm{qi}}^{\mathrm{q}+1} \Psi(\mathrm{q}) \Psi *(\mathrm{q}) \mathrm{dq}$

Using the mean value theorem, we rewrite Eq. (2) As follows:

$\mathrm{p}_{\mathrm{i}}=\left|\Psi_{\mathrm{i}}\left(\tilde{\mathrm{q}}_{\mathrm{i}}\right)\right|^{2} \Delta \mathrm{q}_{\mathrm{i}} ; \mathrm{q}_{\mathrm{i}} \leq \tilde{\mathrm{q}}_{\mathrm{i}} \leq \mathrm{q}_{\mathrm{i}+1}$ where the sum of pi over all spatial intervals is:

$$
\sum_{\mathrm{i}=1}^{\infty} \mathrm{p}_{\mathrm{i}}=\sum_{\mathrm{i}=1}^{\infty}\left|\Psi_{\mathrm{i}}\left(\tilde{\mathrm{q}}_{\mathrm{i}}\right)\right|^{2} \Delta \mathrm{q}_{\mathrm{i}}=1
$$

Using Eq. (3) we get:

$\mathrm{p}_{\mathrm{i}} \log \mathrm{p}_{\mathrm{i}}=\left|\Psi_{\mathrm{i}}\right|^{2}\left[\log \left(\left|\Psi_{\mathrm{i}}\right|^{2}\right)+\log \left(\Delta \mathrm{q}_{\mathrm{i}}\right)\right] \Delta \mathrm{q}_{\mathrm{i}}$

Now we take the following limit of (5):

$\Delta \mathrm{q}_{\mathrm{i}} \rightarrow 0 ; \Delta \mathrm{q}_{\mathrm{i}} \rightarrow \mathrm{dq}$

As a result, Eq. (5) yields:

$\mathrm{p}_{\mathrm{i}} \log \mathrm{p}_{\mathrm{i}} \rightarrow|\Psi(\mathrm{q})|^{2}\left[\log \left(|\Psi(\mathrm{q})|^{2}\right)-1\right] \mathrm{dq}$

and the equation (1) becomes respectively:

$\rho_{\mathrm{I}}=-|\Psi(\mathrm{q})|^{2}\left(\log \left(|\Psi(\mathrm{q})|^{2}\right)-1\right)$

Therefore we narrow our discussion even more by restricting it with continuous spectra. In a 3-D case the integration in Eq. (7) is carried over a spatial volume $\mathrm{d}^{3} \mathrm{q}$. This means that the expression:

$\rho_{\mathrm{I}}=-|\Psi(\mathrm{q})|^{2}\left(\log |\Psi(\mathrm{q})|^{2}-1\right)$ density

can be interpreted as the volume information

$\rho_{\mathrm{I}}=\mathrm{dI} / \mathrm{dV}$

We find its time variation: 


$$
\begin{aligned}
\frac{\partial \rho_{1}}{\partial_{\mathrm{t}}} & =-\frac{\partial}{\partial_{\mathrm{t}}}\left[|\Psi(\mathrm{q})|^{2}\left(\log |\Psi(\mathrm{q})|^{2}-1\right)\right] \\
& =-\log |\Psi|^{2} \frac{\partial|\Psi|^{2}}{\partial \mathrm{t}}
\end{aligned}
$$

If we use the continuity equation for the probability density ${ }^{[3]}$ :

$\frac{\partial|\Psi|^{2}}{\partial \mathrm{t}}=-\frac{\hbar}{2 \mathrm{im}} \operatorname{div}\left[|\Psi|^{2} \nabla\left(\log \frac{\Psi}{\Psi^{*}}\right)\right]$

Then we obtain from Eq. (10):

$\frac{\partial \rho_{\mathrm{I}}}{\partial \mathrm{t}}=\frac{\hbar}{2 \mathrm{im}} \operatorname{div}\left[|\Psi|^{2} \nabla\left(\log \frac{\Psi}{\Psi^{*}}\right)\right] \log |\Psi|^{2}$

$\equiv \operatorname{div}\left[|\Psi|^{2} \log |\Psi|^{2} \frac{\hbar}{2 \mathrm{im}} \nabla\left(\log \frac{\Psi}{\Psi^{*}}\right)\right]$

$-\frac{\hbar}{2 \mathrm{im}} \nabla\left(\log \frac{\Psi}{\Psi^{*}}\right) \square \nabla|\Psi|^{2}$

Inserting the definition of the information density rI, Eq. (8), in the first term on the right hand side of Eq. (11) and denoting the probability density $|\psi| 2=r$, we obtain:

$$
\begin{aligned}
& \frac{\partial \rho_{\mathrm{I}}}{\partial \mathrm{t}}=-\operatorname{div}\left[\frac{\hbar}{2 \mathrm{im}}\left(\rho_{\mathrm{I}}-\rho\right) \nabla\left(\log \frac{\Psi}{\Psi^{*}}\right)\right] \\
& -\frac{\hbar}{2 \mathrm{im}} \nabla\left(\log \frac{\Psi}{\Psi^{*}}\right) \square|\Psi|^{2}
\end{aligned}
$$

Let us consider the transition to the classical case by representing the wave function as follows ${ }^{[3]}$ :

$\Psi=\operatorname{Re}^{\mathrm{i} S / \hbar}$

Using (13) in (12), we get:

$\frac{\partial \rho_{\mathrm{I}}}{\partial \mathrm{t}}=-\operatorname{div}\left[\frac{\nabla \mathrm{S}}{\mathrm{m}}\left(\rho_{\mathrm{I}}-\mathrm{R}^{2}\right)\right]-\frac{\nabla \mathrm{S}}{\mathrm{m}} \bullet \nabla \mathrm{R}^{2}$

In the classical limit $\hbar \mathrm{t}_{\mathrm{c}} / \mathrm{mL}_{\mathrm{c}} \rightarrow 0$ (where $\mathrm{L}_{\mathrm{c}}$ is a characteristic length and $t_{c}$ is the characteristic time) phase $\mathrm{S}$ becomes a classical action. As a result, $\nabla \mathrm{S} / \mathrm{m}=\overrightarrow{\mathrm{v}}$, that is a classical particle velocity. This means that in this limit Eq. (14) yields:

$\frac{\partial \rho_{\mathrm{I}}}{\partial \mathrm{t}}=-\operatorname{div}\left[\overrightarrow{\mathrm{v}}\left(\rho_{\mathrm{I}}-\mathrm{R}^{2}\right)\right]-\overrightarrow{\mathrm{v}} \bullet \nabla \mathrm{R}^{2}$
If we integrate (15) over a volume $\mathrm{V}$, use the definition of the information entropy (9), and Gauss's theorem, we obtain:

$\frac{\partial}{\partial t} \int \rho_{I} d V=\frac{\partial I}{\partial t}=-\oint\left(\rho_{I}-R^{2}\right) \vec{V} \square d \vec{A}-\int \vec{v} \square \nabla R^{2} d V$

By taking the boundary of the volume $\mathrm{V}$ to infinity (where $\vec{v}=0$ ) and assuming that $R_{q \rightarrow \infty} \rightarrow 0$, we obtain from (16):

$\frac{\partial I}{\partial t}=-\int \vec{v} \square \nabla R^{2} d V$

This means that in a transition from a quantum to classical regime the information entropy I [defined in the above narrow sense, Eq. (7)] is not conserved. Instead, if the classical velocity is in the direction of an increase of probability density, the information entropy decreases. Inversely, if the classical velocity is in the direction of a decreasing probability density, the information density increases.

These results are in agreement with the meaning of the information entropy as a number of states accessible to a system. In a transition to a classical regime this number drastically decreases, thus signaling a decrease in information entropy. Quite in agreement with that, and from another point of view, the less probable states carry more information than the more probable states. One can interpret these results as a statement that a classical regime has less degrees of freedom than its quantum counterpart. This is definitely true when there is such a counterpart.

As we have already stated earlier, the above definition of information entropy is not fully appropriate for quantum mechanics because it does not take into account the information carried by a quantum phase. This serves as a strong indication that in the quantum region one needs to use another, more general, the definition of information entropy, not necessarily associated with the qubits, which will account for the information associated with the quantum phase.

\section{ACKNOWLEDGMENT}

The author indebted to the late V. Panico and C. W ulfman for the illuminating discussions of the results.

\section{REFERENCES}

1. Landau, L. and E. Lifshitz, 1980. Statistical Physics. Pergamon Press. 
2. Cerf, N. and C. Adams, ------. Negative Entropy in quantum Information Theory. quantph/ 9610005.
3. Landau, L. and E. Lifshitz, 1974. Quantum Mechanics. Pergamon Press. 